International Journal of Modern Physics A, (c) World Scientific Publishing Company

\title{
NEW MESUREMENTS OF NUCLEON STRUCTURE FUNCTIONS FROM CCFR/NuTeV
}

A. Bodek, ${ }^{7}$ U. K. Yang, ${ }^{7}$ T. Adams, ${ }^{4}$ A. Alton, ${ }^{4}$ C. G. Arroyo, ${ }^{2}$ S. Avvakumov,${ }^{7}$ L. de Barbaro,${ }^{5}$ P. de Barbaro, ${ }^{7}$ A. O. Bazarko, ${ }^{2}$ R. H. Bernstein,${ }^{3}$ T. Bolton, ${ }^{4}$ J. Brau, ${ }^{6}$ D. Buchholz,${ }^{5}$ H. Budd,${ }^{7}$ L. Bugel,${ }^{3}$ J. Conrad,${ }^{2}$ R. B. Drucker, ${ }^{6}$ B. T. Fleming, ${ }^{2}$ J. A. Formaggio, ${ }^{2}$ R. Frey,${ }^{6}$ J. Goldman, ${ }^{4}$ M. Goncharov, ${ }^{4}$ D. A. Harris,${ }^{7}$ R. A. Johnson, ${ }^{1}$ J. H. Kim,${ }^{2}$ B. J. King, ${ }^{2}$ T. Kinnel, ${ }^{8}$ S. Koutsoliotas, ${ }^{2}$ M. J. Lamm, ${ }^{3}$ W. Marsh,${ }^{3}$ D. Mason, ${ }^{6}$ K. S. McFarland, ${ }^{7}$ C. McNulty, ${ }^{2}$ S. R. Mishra, ${ }^{2}$ D. Naples, ${ }^{4}$ N. Suwonjandee, ${ }^{1}$ P. Nienaber, ${ }^{3}$ A. Romosan,${ }^{2}$ W. K. Sakumoto,${ }^{7}$ H. Schellman, ${ }^{5}$ F. J. Sciulli, ${ }^{2}$ W. G. Seligman, ${ }^{2}$ M. H. Shaevitz, ${ }^{2}$ W. H. Smith,${ }^{8}$ P. Spentzouris, ${ }^{2}$ E. G. Stern, ${ }^{2}$ M. Vakili, ${ }^{1}$ A. Vaitaitis,${ }^{2}$ J. Yu, ${ }^{3}$ G. P. Zeller, ${ }^{5}$ and E. D. Zimmerman ${ }^{2}$

(Presented at DPF2000 by Arie Bodek for the CCFR/NuTeV Collaboration - UR-1615)

1 Univ. of Cincinnati, Cincinnati, OH 45221; ${ }^{2}$ Columbia University, New York, NY 10027

${ }^{3}$ Fermilab, Batavia, IL $60510^{4}$ Kansas State University, Manhattan, KS 66506

${ }^{5}$ Northwestern University, Evanston, IL 60208; 6 Univ. of Oregon, Eugene, OR 97403

7 Univ. of Rochester, Rochester, NY 14627; ${ }^{8}$ Univ. of Wisconsin, Madison, WI 53706

We report on the extraction of the structure functions $F_{2}$ and $\Delta x F_{3}=x F_{3}^{\nu}-x F_{3}^{\bar{\nu}}$ from CCFR $\nu_{\mu}$-Fe and $\bar{\nu}_{\mu}$-Fe differential cross sections. The extraction is performed in a physics model independent (PMI) way. This first measurement for $\Delta x F_{3}$, which is useful in testing models of heavy charm production, is higher than current theoretical predictions. Within $5 \%$ the $F_{2}$ (PMI) values measured in $\nu_{\mu}$ and $\mu$ scattering are in agreement with the predictions of Next-to-Leading-Order PDFs (using massive charm production schemes), thus resolving the long-standing discrepancy between the two measurements.

Deep inelastic lepton-nucleon scattering experiments have been used to determine the quark distributions in the nucleon. However, the quark distributions determined from $\mu$ and $\nu$ experiments were found to be different at small values of $x$, because of a disagreement in the extracted structure functions. Here, we report on a measurement of differential cross sections and structure functions from CCFR $\nu_{\mu}$-Fe and $\bar{\nu}_{\mu}$-Fe data. We find that the neutrino-muon difference is resolved by extracting the $\nu_{\mu}$ structure functions in a physics model independent way.

The sum of $\nu_{\mu}$ and $\bar{\nu}_{\mu}$ differential cross sections for charged current interactions on an isoscalar target is related to the structure functions as follows:

$F(\epsilon) \equiv\left[\frac{d^{2} \sigma^{\nu}}{d x d y}+\frac{d^{2} \sigma^{\bar{\nu}}}{d x d y}\right] \frac{(1-\epsilon) \pi}{y^{2} G_{F}^{2} M E_{\nu}}=2 x F_{1}[1+\epsilon R]+\frac{y(1-y / 2)}{1+(1-y)^{2}} \Delta x F_{3}$

Here $G_{F}$ is the Fermi weak coupling constant, $M$ is the nucleon mass, $E_{\nu}$ is the incident energy, the scaling variable $y=E_{h} / E_{\nu}$ is the fractional energy transferred to the hadronic vertex, $E_{h}$ is the final state hadronic energy, and $\epsilon \simeq 2(1-y) /(1+$ $\left.(1-y)^{2}\right)$ is the polarization of the virtual $W$ boson. The structure function $2 x F_{1}$ is expressed in terms of $F_{2}$ by $2 x F_{1}\left(x, Q^{2}\right)=F_{2}\left(x, Q^{2}\right) \times \frac{1+4 M^{2} x^{2} / Q^{2}}{1+R\left(x, Q^{2}\right)}$, where $Q^{2}$ is the square of the four-momentum transfer to the nucleon, $x=Q^{2} / 2 M E_{h}$ (the Bjorken scaling variable) is the fractional momentum carried by the struck quark, 
and $R=\frac{\sigma_{L}}{\sigma_{T}}$ is the ratio of the cross-sections of longitudinally- to transverselypolarized $W$ bosons. The $\Delta x F_{3}$ term, which in leading order $\simeq 4 x(s-c)$, is not present in the $\mu$-scattering case. In addition, in a $\nu_{\mu}$ charged current interaction with $s($ or $\bar{c}$ ) quarks, there is a threshold suppression originating from the production of heavy $c$ quarks in the final state. For $\mu$-scattering, there is no suppression for scattering from $s$ quarks, but more suppression when scattering from $c$ quarks since there are two heavy quarks $(c$ and $\bar{c})$ in the final state.

In previous analyses of $\nu_{\mu}$ data, structure functions were extracted by applying a slow rescaling correction to correct for the charm mass suppression in the final state. In addition, the $\Delta x F_{3}$ term (used as input in the extraction) was calculated from a leading order charm production model. These resulted in a physics model dependent (PMD) structure functions. In the new analysis reported here, slow rescaling corrections are not applied, and $\Delta x F_{3}$ and $F_{2}$ are extracted from twoparameter fits to the data. We compare the values of $\Delta x F_{3}$ to various charm production models. The extracted physics model independent (PMI) values for $F_{2}^{\nu}$ are then compared with $F_{2}^{\mu}$ within the framework of NLO models for massive charm production.
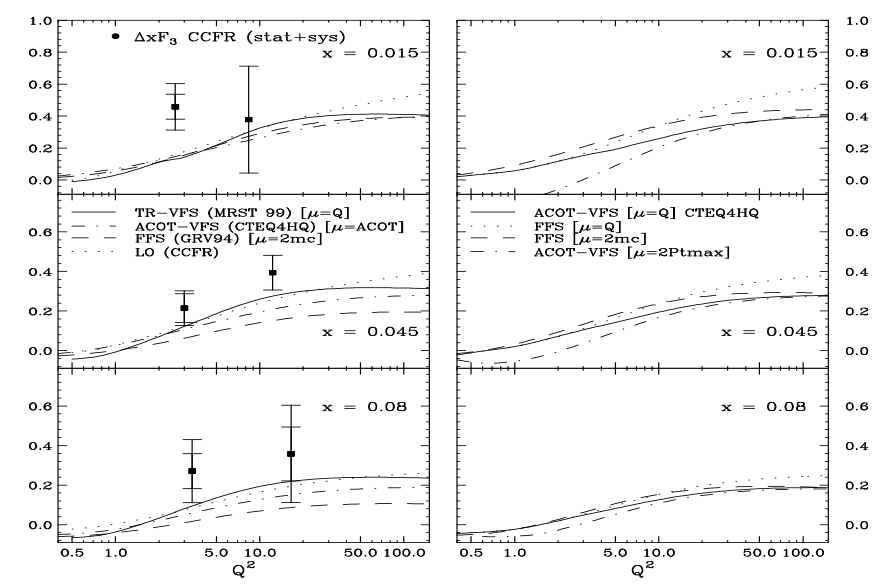

Fig. 1. $\Delta x F_{3}$ data as a function of $x$ compared with various schemes for massive charm production: RT-VFS(MRST), ACOT-VFS(CTEQ4HQ), FFS(GRV94), and LO(CCFR), a leading order model with a slow rescaling correction (left); Also shown is the sensitivity of the theoretical calculations to the choice of scale (right).

Because of the limited statistics, we use large bins in $Q^{2}$ in the extraction of $\Delta x F_{3}$ with bin centering corrections. Figure 1 shows the extracted values of $\Delta x F_{3}$ as a function of $x$, including both statistical and systematic errors, compared to various theoretical methods for modeling heavy charm productions within a QCD framework. Figure 1 (right) also shows the sensitivity to the choice of scale. With reasonable choices of scale, all the theoretical models yield similar results. However, at low $Q^{2}$ our $\Delta x F_{3}$ data are higher than all the theortical models. The difference between data and theory may be due to an underestimate of the strange sea at low $Q^{2}$, or from missing NNLO terms. 
Values of $F_{2}$ (PMI) for $x<0.1$ are extracted from two-parameter fits to the $y$ distributions. In the $x>0.1$ region, the contribution from $\Delta x F_{3}$ is small and the extracted values of $F_{2}$ are insensitive to $\Delta x F_{3}$. Therefore, we extract values of $F_{2}$ with an input value of $R$ and with $\Delta x F_{3}$ constrained to the TR-VFS(MRST) predictions. As in the case of the two-parameter fits for $x<0.1$, no corrections for slow rescaling are applied. Fig. 2 shows our $F_{2}$ (PMI) measurements divided by the predictions from the TR-VFS(MRST) theory. Also shown are $F_{2}^{\mu}$ and $F_{2}^{e}$ divided by the theory predictions. In the calculation of the QCD TR-VFS(MRST) predictions, we have also included corrections for nuclear effects, target mass and higher twist corrections at low values of $Q^{2}$. As seen in Fig. 2, within $5 \%$ both the neutrino and muon structure functions are in agreement with the TR-VFS(MRST) predictions, and therefore in agreement with each other. thus resolving the long-standing discrepancy between the two sets of data. A comparison using the ACOT-VFS(CTEQ4HQ) predictions yields similar results. Note that in the previous analysis of the CCFR data, the extracted values of $F_{2}$ (PMD) at the lowest $x=0.015$ and $Q^{2}$ bin were up to $20 \%$ higher than both the NMC data and the predictions of the light-flavor MRSR2 PDFs. More details on this work can be found in UR- 1586 (hep-ex/0009041, submitted to Phys. Rev. Lett. 9/00), and in U. K. Yang, Ph.D. Thesis, Univ. of Rochester (UR-1583, in preparation).
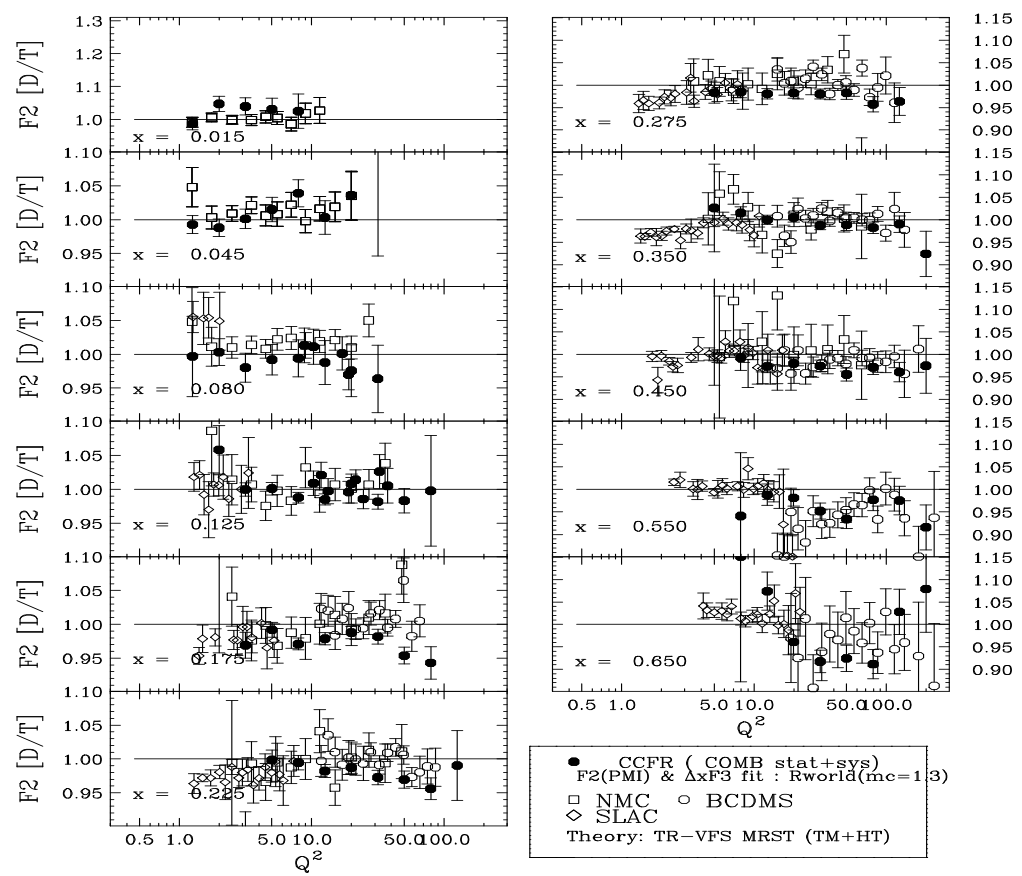

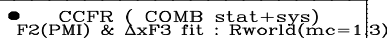
$\checkmark$ NMC $\triangle$ BCDMS

Theory: TR-VFS MRST (TM+HT)

Fig. 2. The ratio (data/theory) of the $F_{2}^{\nu}$ (PMI) data divided by the predictions of TRVFS(MRST) (with target mass and higher twist corrections). Both statistical and systematic errors are included. Also shown are the ratios of the $F_{2}^{\mu}$ (NMC,BCDMS) and $F_{2}^{e}$ (SLAC) to the TR-VFS(MRST) predictions. 